\title{
Emerging Approaches in Molecular Profiling Affecting Oncology Drug Discovery
}

\author{
S.H. FRIEND \\ Merck Research Laboratories, West Point, Pennsylvania 19486; Rosetta Inpharmatics, LLC, a wholly owned \\ subsidiary of Merck \& Co., Inc., Seattle, Washington 98109
}

\begin{abstract}
The purpose of this paper is to provide some perspectives on whether we are at a tipping point in understanding oncology and oncology drug discovery. It describes how model organisms have prepared us for more efficient drug discovery, lessons that are in use today. It provides examples of the emerging integration of biomarkers in patient care. It also details how over the next several years the processes of carrying out target identification and identifying responders to drugs will become more and more similar. In conclusion, a discussion is provided about who can do what to link the various components of this information-rich drug discovery process together.
\end{abstract}

If we turn the clock back to 1995 , we find already increased numbers of specific molecular targets. Several lines of evidence were able to show specific mechanisms of actions surrounding specific targeted therapies, and there was an emerging understanding of the specific risks that came from genetic alterations. Despite this, there was minimal knowledge on why any of the existing therapies in cancer were working in specific patients. In 1995 I was fortunate to have an in-depth discussion with Harold Varmus, who was eloquent in pointing out the difference between probing the pathophysiology of disease and trying to change therapeutic options for patients. The outcome of this discussion was a remarkable year in which I took a sabbatical going in and out of pharmaceutical companies with the express purpose of better understanding the diversity and how large pharma and biotech companies carried out drug discovery. Four main lessons came out of this year. First, it was clear that the methods used in a general sense to identify the right targets were primarily narrowly chosen. Such efforts were mainly guided by interest in specific genes that had been in vogue. Second, the efforts needed to move early compounds through to drugs required primarily brute force to optimize the ontarget effects of these compounds. This required the significant use of resources, not only time, but also cost. Third, the ability in oncology to identify the right subpopulations for the right drugs is done primarily empirically. As is well known in the drug discovery world, it is not uncommon for more than ten clinical trials to be performed in oncology before a respectable signal of response can be found. The last lesson was that the critical design for combination therapies that are the basis of much of the therapy given to patients currently is one of "trial and error." Coming out of this sabbatical year, it was easy to commit to more efficient drug discovery. At this time, I was fortunate to link up with Lee Hartwell, who was a key proponent for the use of genetics to more rationally design drugs. Thus began a period of collaboration of work based initially in yeast to develop models to increase the probability of success in developing drugs. The outcome of this work involves the nonbiased detection of targets and the nonbiased identification of individuals who might be likely to respond.

Working with Lee Hartwell, it became evident that in the long run the most valuable tool to help guide drug discovery might be an "ideal detector" that allowed one to simultaneously monitor the function of all proteins within a given cell. To develop such a detector, one would need tens of thousands of independent reporters to detect changes within the cell. Second, it would be important to build a large database of known patterns. And third, one would need to be able to compare unknown patterns to large compendia of known patterns. The most obvious technology available at the time to carry this out was provided by the gene expression arrays that were beginning to be commonly used in the mid-1990s. The early experiments that we carried out to understand the limits of this method involved developing probes that could detect the levels of each of the genes in the yeast, Saccharomyces cerevisiae. It also required building a compendium of knockout strains of yeast that were knocked out either as single copies of mutants, or mutants where both alleles of any given gene were knocked out. By linking a large international consortium of yeast laboratories, it was possible to build this compendium of mutants and then to profile hundreds of mutants to compile compound and mutant yeast profiles. As shown in Figure 1, drugs such as lovastatin clustered with gene disruptions such as hmg $1 / 2$ and drug signatures of the compound tunicamycin were closely linked to the signature for the disruption of the gas1 gene. This work, carried out by Tim Hughes and others at Rosetta Inpharmatics, showed that many of the boundary conditions inside a simple organism were sufficient to "decode" the mechanism of action related to drugs by comparing it to known genetic alterations (Hughes et al. 2000).

A more difficult test of the compendium approach was to determine whether it was possible to use expression pro- 


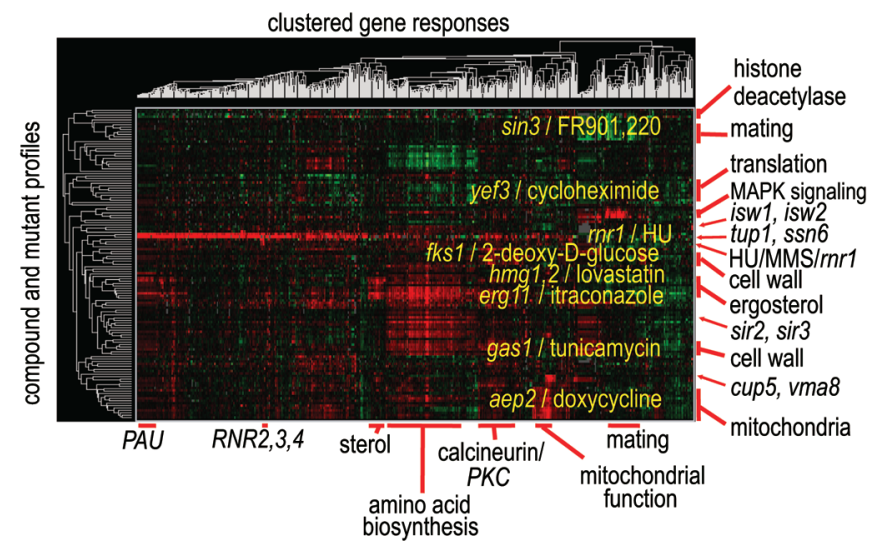

Figure 1. Two-dimensional agglomerative hierarchical clustering of 127 experiments and 568 genes, selected to include only experiments with 2 or more genes up- or down-regulated greater than threefold, and significant at $p \leq 0.01$; and only genes that are up- or down-regulated at greater than threefold, and at $p \leq 0.01$, in 2 or more experiments. (PAU) PAU gene family; $(S / C)$ stress and carbohydrate metabolism; $(A A)$ amino acid biosynthesis; $(P K C)$ responsive to protein kinase C. (Reprinted, with permission, from Hughes et al. 2000 [C Elsevier].) files in various mutant cells to build signaling pathways. An article published by Chris Roberts and coworkers in 2000 showed that combining genetics and expression profiling allowed one to reveal MAPK pathway cross talk. These early experiments gave strong evidence that under some conditions, the diversity of information captured from yeast was able to inform the mechanism of action of compounds (Roberts et al. 2000).

At the same time, it was possible to answer other questions related to the context specificity of cancer drugs by doing other experiments in yeast. In particular, we were interested in determining whether it was possible to delineate the mechanisms of action of compounds by looking at the effects that compounds had in strains of yeast particular genetic mutations. Work published by Phillip Szankasi, Chris Roberts, Andrew Murray, and others in 1997 showed the ability to determine the mechanism of different commonly used chemotherapeutic agents by this strategy. Two examples are worth noting. Treating strains of yeast with a large range of mutations in various pathways showed a high sensitivity to cisplatin in yeast that had defects in the genes rad6 and rad18 that controlled the pathway for repairing the damage to cisplatin. Similarly, experiments looking at the sensitivity of various mutant strains to mitoxantrone showed a high degree of sensitivity when yeast had defects in $\operatorname{rad} 50$, rad51, and rad52 that made up the double-strand-break repair pathway (Hartwell and Weinert 1989).

The other question answered in yeast was whether one could carry out nonbiased genome-wide screens to identify druggable targets that could selectively kill cells harboring defects found in human tumors. During the years 1996-1998, a large group of scientists including Eric Foss, Chris Roberts, Julian Simon, John Lamb, Jenya Kroll, and Brian Thornton at the Seattle Project based at the Fred Hutchinson Cancer Research Center began to carry out synthetic lethal screens. One of these started with a defect in mismatched repair by knocking out the mlh1 gene. This screen revealed that alterations in polymerase $\delta$, polymerase $\varepsilon$, and $\operatorname{rnr} 1$ were all synthetic lethal with defects in mlh1. A second screen was designed around defects in the
ATM pathway used as the primary mutation disruption of the mec1 gene. In the mec1 synthetic lethal screen, it was possible to show synthetic lethal alleles that ranged from rnr1, rnr2, and rnr4 through cdc8, cdc17, and cdc21. This screen also identified PRI1 (DNA primates subunit) and $\operatorname{rad} 27$, which is a 5' flap endonuclease.

All of the above experiments involved with profiling known and unknown patterns, searching for mechanisms of action using known drugs and specific mutations, and finally, the synthetic lethal screens, were carried out in yeast because the organism was genetically valuable and its complete sequence was known. These experiments helped determine the boundary conditions for the mammalian experiments we carry out today, but they only gave distant clues as to the specific realities of such pathways in human systems. The transition from work in yeast model systems to experiments in man represented one of the major missions of Rosetta Inpharmatics. The rest of the experiments to be described here involve more recent efforts to carry out similar experiments in mammalian cells.

Some of the early experiments that identified differences in the behavior of human tumors came from a large collaboration set up with Rene Bernards and Laura Van't Veer at the Netherlands Cancer Institute. Working with samples that had been carefully collected over many years and their accompanying medical records, it was possible to delineate patients with breast cancer who had progressed to distant metastasis rapidly as opposed to another set of patients who had had prolonged survival without distant metastasis. In 2002, we published gene signatures that allowed us to distinguish these different sets of patients using full genome expression profiling (Van't Veer et al. 2002). This work and similar experiments by many other investigators have enabled scientists to routinely search for expression profiles that can be linked to the natural history of various cancers and, more importantly, to identify the response to particular drugs. The early concept of building a compendium of known patterns to identify the biology of unknown samples has become commonplace in many oncology treatment protocols. Some of the more exciting recent work has in- 
volved a closer linkage between specific clinical parameters and molecular signatures. One example that we recently published involved identifying a set of patients who had high levels of estrogen receptors in their breast cancers but who had an extremely poor outcome that could be predicted by monitoring expression profiling (Dai et al. 2005). It is likely that this combination of clinical and molecular profiling clues will be commonly integrated so that the right patient is able to get the right drug at the right time.

The counterpart to the original unbiased full-genome screens that were carried out in yeast have also been carried out in mammalian systems. Working with a number of investigators at Cold Spring Harbor Laboratory, at the Salk Institute, and at biotech companies such as Alnylam, we have developed siRNA oligos and shRNA vectors to disrupt the expression of specific genes in mouse and human cells. The siRNA libraries that we have been using involve developing pools of siRNAs that in each well have three different siRNAs for a particular gene. We have developed such siRNA pools for 22,116 human genes, and we also have developed siRNAs that inhibit over 200 micro RNAs. Within the Automated Biotechnology Center in North Wales, Pennsylvania at Merck Research Laboratories and at Rosetta Inpharmatics in Seattle, Washington, we have developed automated robotics capabilities that allow us to carry out primary synthetic lethal screens over a period of weeks. These fully automated siRNA synthetic lethal screens involve plating cells on day 1 , transfecting siRNAs on day 2 , treating the cells with drugs and adding sensitizers the next day, and following the growth of various cells. We have found that it is necessary to have highly consistent transfection efficiencies. We have found it important to have a consistent sensitization of reference genes in the presence of sensitizer agents. We also have found it important to test for siRNA library integrity again using internal controls. As shown in Figure 2, it is possible to carry out a gemcitabine siRNA high-throughput screen in HeLa cells according to the methods just mentioned. It was helpful to identify primary hits at both low and high concentrations of gemcitabine and also to look for the overlap of genes that sensitize both low and high concentrations of gemcitabine. These selective primary hits were then retested, and a subset of confirmed primary hits was obtained. Thirty-two genes were found to confirm as primary hits at low concentrations of gemcitabine; 180 genes were confirmed to hit at high concentrations of gemcitabine. More importantly, 127 genes were confirmed to produce synthetic lethality with gemcitabine at both low and high concentrations. Preliminary analysis of the confirmed hits from the HeLa cell gemcitabine screen indicated enrichment of genes in a number of different pathways. We have found genes that are involved with microtubule spindle and centrosome assembly. Preliminary analysis also suggests genes involved with proteosome stability and RNA metabolism.

By carrying out synthetic lethal screens with both compounds and RNAs, we have identified situations where both siRNAs and small molecular combinations will sensitize cells that are either wild-type or defective for the

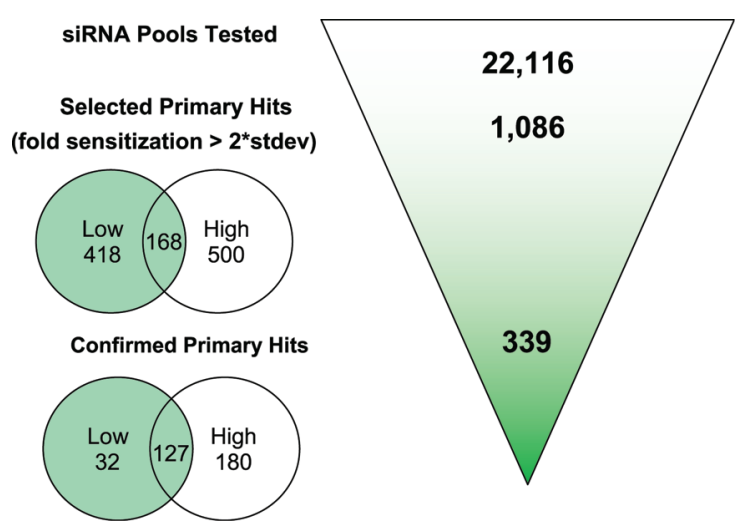

Figure 2. Summary of the HeLa/gemcitabine siRNA highthroughput screen results. Selected hits are highlighted where there is a sensitization greater than two standard deviations.

p53 gene. For the primary screens involving the compounds, we began with approximately 1.5 million compounds, and by filtering those for confirmed hits and for hits that exhibited greater than 1.5 -fold sensitivity for $\mathrm{p} 53$ cells, we still retained over 1000 compounds. By selecting for compounds that exhibited the greatest dosedependent effect, this set of genes was able to be downselected to 11 compounds. Possibly the most interesting conclusion from these experiments has come from taking these sensitizing compounds and testing them in panels of cell lines from diverse tissues, all of which have been rendered as matched pairs, either having wild-type or disrupted p53 using shRNA vectors to disrupt the p53 status. Such studies have shown that compounds which may provide sensitization in one cell type that is defective for p53 will sometimes be able to provide an increased sensitivity across other cell lines of diverse tissues; whereas other compounds seem to have more limited sensitization that is restricted to particular cell lines. We consider this an important result because it more closely approximates the diversity that is likely to be found in actual patients. In summary, we think that the combination of unbiased syn-

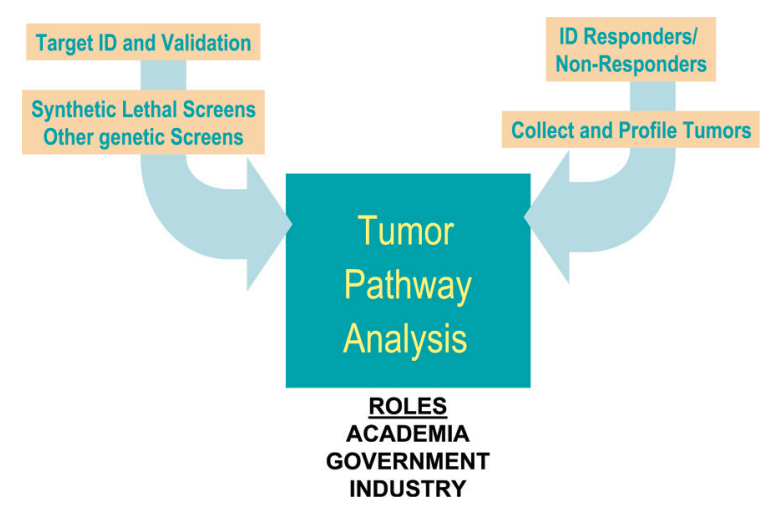

Figure 3. Tumor pathway analysis: Benefit of combining the efforts to identify and validate targets with the information used to identify responders. 
thetic lethal screens to define targets, and unbiased synthetic lethal screens to define compounds, will be valuable, not only for identifying new targets for cancer therapies, but also in better identifying which patients these compounds are most likely to work in.

Over the next 5-10 years, significant work is needed to take these preliminary unbiased screens for new targets and powerful methods for identifying responders in order to affect drug discovery. The good news is that many of these methods are now ready to be used in the broader clinical environment. At the same time, significant issues still need to be worked out related to the roles academia, government, and industry can take in order to most efficiently integrate these new technologies into active patient care. Until recently, the early work in drug discovery involved with identifying targets and validating those targets has been seen as quite separate from the work that is being done by clinicians to collect and profile tumors. As shown in Figure 3, many of the methods that have been used for target ID and validation using profiling and synthetic lethal screens are likely in the future to be used to identify responders and nonresponders. The reason for this is that at the heart of the identification of new targets, and of patients who respond to drugs, is the need for a better understanding of tumor pathway analysis. The major limitation in achieving both these goals is likely to be the tools that are needed to interrogate the information generated by these diverse biological methods.

\section{ACKNOWLEDGMENTS}

We thank Drs. Peter Linsley, Steven Bartz, Aimee Jackson, Michele Cleary, Priya Kunapuli, Namjin Chung, Marc Ferrer, and Berta Strulovici.

\section{REFERENCES}

Dai H., van’t Veer L., Lamb J., He Y.D., Mao M., Fine B.M., Bernards R., van de Vijver M., Deutsch P., Sachs A., Stoughton R., and Friend S.H. 2005. A cell proliferation signature is a marker of extremely poor outcome in a subpopulation of breast cancer patients. Cancer Res. 65: 4059.

Hartwell L.H. and Weinert T.A. 1989. Checkpoints: Controls that ensure the order of cell cycle events. Science 246: 629.

Hughes T.R., Marton M.J., Jones A.R., Roberts C.J., Stoughton R., Armour C.D., Bennett H.A., Coffey H., Dai H., He Y.D., Kidd M.J., King A.M., Meyer M.R., Slade D., Lum P.Y., Stepaniants S.B., Shoemaker D.D., Gachotte D., Chakraburtty K., Simon J., Bard M., and Friend S.H. 2000. Functional discovery via a compendium of expression profiles. Cell 102: 109.

Roberts C.J., Nelson B., Marton M.J., Stoughton R., Meyer M.R., Bennett H.A., He Y., Dai H., Walker W., Hughes T., Tyers M., Boone C., and Friend S.H. 2000. Signaling and circuitry of multiple MAPK pathways revealed by a matrix of global gene expression profiles. Science 287: 873.

Van't Veer L.J., Dai H., Van de Vijver M.J., He Y.D., Hart A.A.M., Mao M., Peterse H.L., Van der Kooy K., Marton M.J., Witteveen A.T., Schreiber G.J., Kerkhoven R.M., Roberts C.J., Linsley P.S., Bernards R., and Friend S.H. 2002. Gene expression profiling predicts clinical outcome of breast cancer. Nature 415: 530 . 


\section{$\$_{\text {CSH\& }}^{\infty} \mathrm{Cold}$ Spring Harbor Symposia SYMPOSIA on Quantitative Biology}

\section{Emerging Approaches in Molecular Profiling Affecting Oncology Drug Discovery}

S.H. FRIEND

Cold Spring Harb Symp Quant Biol 2005 70: 445-448

Access the most recent version at doi:10.1101/sqb.2005.70.028

References This article cites 5 articles, 3 of which can be accessed free at:

http://symposium.cshlp.org/content/70/445.full.html\#ref-list-1

License

Email Alerting Receive free email alerts when new articles cite this article - sign up in

Service the box at the top right corner of the article or click here.

To subscribe to Cold Spring Harbor Symposia on Quantitative Biology go to: http://symposium.cshlp.org/subscriptions 